EUROPEAN JOURNAL OF PURE AND APPLIED MATHEMATICS

Vol. 10, No. 3, 2017, 455-472

ISSN 1307-5543 - www.ejpam.com

Published by New York Business Global

\title{
States on pseudo-BCI algebras
}

\author{
Xiao Long $\mathrm{Xin}^{1, *}$, Yi Jun $\mathrm{Li}^{1}$, Yu Long $\mathrm{Fu}^{2}$ \\ 1 School of Mathematics, Northwest University, Xi'an, China \\ 2 School of Cyber Engnieering, Xidian University, Xi'an, China
}

\begin{abstract}
In this paper, we discuss the structure of pseudo-BCI algebras and get that any pseudoBCI algebra is a union of it's branches. We introduce the notion of local bounded pseudo-BCI algebras and study some related properties. Moreover we define two operations $\wedge_{1}, \wedge_{2}$ in a local bounded pseudo-BCI algebra $A$ and two local operations $\vee_{1}$ and $\vee_{2}$ in $V(a)$ for $a \in M(A)$. We show that in a $\wedge_{1}\left(\wedge_{2}\right)$-commutative local bounded pseudo-BCI algebra $A,\left(V(a), \wedge_{1}, \vee_{1}\right)\left(\left(V(a), \wedge_{2}, \vee_{2}\right)\right)$ forms a lattice for all $a \in M(A)$. We define a Bosbach state on a local bounded pseudo-BCI algebra. Then we give two examples of local bounded pseudo-BCI algebras to show that there is local bounded pseudo-BCI algebras having a Bosbach state but there is some one having no Bosbach states. Moreover we discuss some basic properties about Bosbach states. If $s$ is a Bosbach state of a local bounded pseudo-BCI algebra $A$, we prove that $A / \operatorname{ker}(s)$ is equivalent to an $\mathrm{MV}$-algebra. We also introduce the notion of state-morphisms on local bounded pseudo-BCI algebras and discuss the relations between Bosbach states and state-morphisms. Finally we give some characterization of Bosbach states.
\end{abstract}

2010 Mathematics Subject Classifications: 03G25

Key Words and Phrases: Pseudo-BCI algebra, local bounded, state, state-morphism, MValgebra

\section{Introduction}

BCK/BCI algebras were introduced originally by Iséki in [17] and [18] with a binary operation $*$ modeling the set-theoretical difference. Another motivation is from classical and non-classical propositional calculi modeling logical implications. Such algebras contain as a special subfamily of a family of MV-algebras where some important fuzzy structures can be studied. For more about BCK algebras, see [22].

Pseudo-BCK algebras were originally introduced by Georgescu and Iorgulescu in [13] as algebras with "two differences", a left- and right-difference, instead of one $*$ and with a constant element 0 as the least element. In [12], a special subclass of pseudo-BCK algebras, called Łukasiewicz pseudo-BCK algebras, was introduced and it was shown that

${ }^{*}$ Corresponding author.

Email addresses: xlxin@nwu.edu.cn (X.L. Xin), liyijun2009@126.com (Y.J. Li), ylfu@xidian.edu.cn (Y.L. Fu) 
it is always a subalgebra of the positive cone of some $\ell$-group (not necessarily abelian). The class of Łukasiewicz pseudo-BCKalgebras is a variety whereas the class of pseudoBCKalgebras is not; it is only a quasivariety because it is not closed under homomorphic images. For a guide through the pseudo-BCK algebras realm, see the monograph [16]. In [8], W. A. Dudek and Y. B. Jun introduced the notion of pseudo-BCI algebras as an extension of BCI-algebras, and investigated some properties.

$\mathrm{MV}$-algebras entered into mathematics just 50 years ago due to Chang [3], but the notion of a state for MV-algebras was introduced by Mundici [23] in 1995 as averaging of the truth-value in Łukasiewicz logic. BL-algebras were introduced in the 1990s by Hájek [14] as the equivalent algebraic semantics for its basic fuzzy logic. In [5], authors defined a state-operator and a strong state-operator for a BL-algebra and prove some of their basic properties. L. Z. Liu studied the existence of Bosbach states and Riečan states on finite monoidal t-norm based algebras in [21]. Some examples show that there exist MTL-algebras having no Bosbach states and Riečan states.

In [10], Dvurečenskij introduced measures and states on BCK-algebras, and showed that the set of elements of measure 0 is an ideal, and the corresponding quotient BCKalgebra is commutative with a lifted original measure. Ciungu and Dvurečenskij [4] extended the notions of measures and states presented in Dvurečen-skij and Pulmannová [9] to the case of pseudo-BCK algebras, studied similar properties, and prove that, under some conditions, the notion of a state in the sense of Dvurečenskij and Pulmannová [9] coincides with the Bosbach state.

The aim of this paper is to introduce and study the state theory on local bounded pseudo-BCI algebras. This paper is organized as follows: in Section 2, we recall notions of BCI-algebras and the notion and some properties of pseudo-BCI algebras. In the same time, we discuss the structure of pseudo-BCI algebras and get that any pseudo-BCI algebra is a union of it's branches. In Section 3, we introduce the notion of local bounded pseudoBCI algebras and study some related properties. In Section 4, we define a Bosbach state on a local bounded pseudo-BCI algebra. Then we give two examples of local bounded pseudo-BCI algebras to show that there is local bounded pseudo-BCI algebras having a Bosbach state but there is some one having no Bosbach states. Moreover we discuss some of their basic properties. We discuss the relation between local bounded pseudoBCI algebras and $M V$-algebras. We also introduce the notion of state-morphisms on local bounded pseudo-BCI algebras and discuss the relations between Bosbach states and state-morphisms. Finally we give some characterization on Bosbach states.

\section{Pseudo-BCI algebras}

Recall that a BCI-algebra is an algebra $(X, *, 0)$ of type $(2,0)$ satisfying the following axioms: for every $x, y, z \in X,(1)((x * y) *(x * z)) *(z * y)=0,(2)(x *(x * y)) * y=0$, (3) $x * x=0$, (4) $x * y=0$ and $y * x=0$ imply $x=y$.

For any BCI-algebra $X$, the relation $\leq$ defined by $x \leq y$ if and only if $x * y=0$ is a partial order on $X$. A nonempty subset $I$ of a BCI-algebra $X$ is called a BCI- ideal of $X$ if it satisfies (1) $0 \in I$, (2) For all $x, y \in X, x * y \in I, y \in I \Rightarrow x \in I$. 
We recall the notion and some properties of pseudo-BCI algebras.

Definition 1. [19] A pseudo-BCI algebras is a structure $\mathbb{A}=(A, \leq, *, \circ, 0)$, where $\leq$ is a binary relation on $A, *$ and $\circ$ are binary operations on $A$ and " 0 " is an element of $A$, satisfying, for all $x, y, z \in A$,

$\left(I_{1}\right)(x * y) \circ(x * z) \leq z * y,(x \circ y) *(x \circ z) \leq z \circ y$.

$\left(I_{2}\right) x *(x \circ y) \leq y, x \circ(x * y) \leq y$.

$\left(I_{3}\right) x \leq x$.

$\left(I_{4}\right) x \leq y$ and $y \leq x$ imply $x=y$.

$\left(I_{5}\right) x \leq y$ iff $x * y=0$ iff $x \circ y=0$.

Definition 2. [13] A pseudo-BCK algebra is a structure $\mathbb{A}=(A, \preceq, \rightarrow, \rightsquigarrow, 1)$ where $\preceq$ is a binary relation on $A$, and $\rightarrow$ and $\rightsquigarrow$ are binary operations on $A$ and 1 is an element of $A$ satisfying, for all $x, y, z \in A$, the axioms:

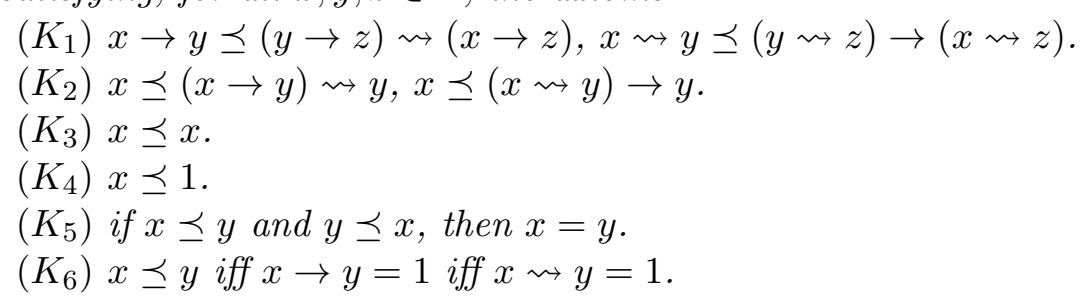

Remark 1. (1) A pseudo-BCK algebra $\mathbb{A}=(A, \preceq, \rightarrow \rightsquigarrow, 1)$ can be seen a pseudo-BCI algebra $\mathbb{A}=(A, \leq, *, \circ, 0)$ if $x \rightarrow y=y * x, x \rightsquigarrow y=y \circ x, 1=0$ and $x \preceq y$ iff $y \leq x$ for all $x, y \in A$.

(2) A pseudo-BCI algebra is a BCI algebra if $*=0$.

(3) The relation $\leq$ is a partial order on a pseudo-BCI algebra $A$.

Now we give two pseudo-BCI algebras which are not pseudo-BCK algebras.

Example 1. Let $A=\{0, u, v, w, t, 1, a, b\}$. The order of the elements in $A$ is as the following Hasse diagram:
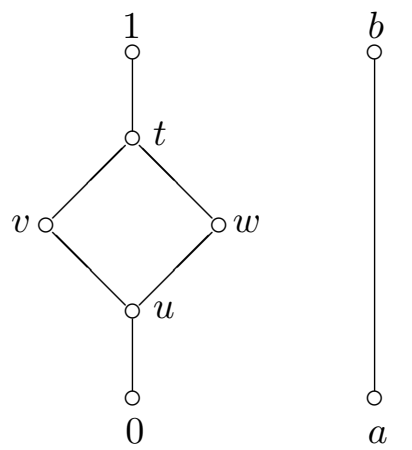

Now the operations $*$ and $\circ$ are defined by Tables 2.1 and 2.2, respectively. Simple calculations show that $(A, \leqslant, *, \circ, 0)$ is a pseudo-BCI algebra.

Example 2. Let $A=\{0, x, y, z, 1, a, b\}$ in which the order of elements in $A$ is as the following Hasse diagram: 


\begin{tabular}{|c|c|c|c|c|c|c|c|c|}
\hline$*$ & 0 & $\mathrm{u}$ & $\mathrm{v}$ & $\mathrm{w}$ & $\mathrm{t}$ & 1 & $\mathrm{a}$ & $\mathrm{b}$ \\
\hline 0 & 0 & 0 & 0 & 0 & 0 & 0 & $\mathrm{a}$ & $\mathrm{a}$ \\
\hline $\mathrm{u}$ & $\mathrm{u}$ & 0 & 0 & 0 & 0 & 0 & $\mathrm{a}$ & $\mathrm{a}$ \\
\hline $\mathrm{v}$ & $\mathrm{v}$ & $\mathrm{v}$ & 0 & $\mathrm{v}$ & 0 & 0 & $\mathrm{a}$ & $\mathrm{a}$ \\
\hline $\mathrm{w}$ & $\mathrm{w}$ & $\mathrm{w}$ & $\mathrm{w}$ & 0 & 0 & 0 & $\mathrm{a}$ & $\mathrm{a}$ \\
\hline $\mathrm{t}$ & $\mathrm{t}$ & $\mathrm{t}$ & $\mathrm{w}$ & $\mathrm{t}$ & 0 & 0 & $\mathrm{a}$ & $\mathrm{a}$ \\
\hline 1 & 1 & 1 & 1 & 1 & 1 & 0 & $\mathrm{a}$ & $\mathrm{a}$ \\
\hline $\mathrm{a}$ & $\mathrm{a}$ & $\mathrm{a}$ & $\mathrm{a}$ & $\mathrm{a}$ & $\mathrm{a}$ & $\mathrm{a}$ & 0 & 0 \\
\hline $\mathrm{b}$ & $\mathrm{b}$ & $\mathrm{b}$ & $\mathrm{b}$ & $\mathrm{b}$ & $\mathrm{b}$ & $\mathrm{a}$ & 1 & 0 \\
\hline
\end{tabular}

Tables 2.1

\begin{tabular}{|c|c|c|c|c|c|c|c|c|}
\hline o & 0 & u & v & w & t & 1 & a & b \\
\hline 0 & 0 & 0 & 0 & 0 & 0 & 0 & a & a \\
\hline u & u & 0 & 0 & 0 & 0 & 0 & a & a \\
\hline v & v & v & 0 & v & 0 & 0 & a & a \\
\hline w & w & w & w & 0 & 0 & 0 & a & a \\
\hline t & t & t & t & v & 0 & 0 & a & a \\
\hline 1 & 1 & 1 & 1 & 1 & 1 & 0 & a & a \\
\hline a & a & a & a & a & a & a & 0 & 0 \\
\hline b & b & b & b & b & b & a & 1 & 0 \\
\hline
\end{tabular}

Tables 2.2

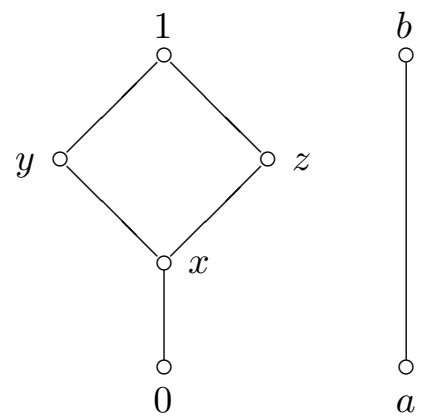

Let the operations $*$, o be given by the following Tables 2.3 and 2.4.

\begin{tabular}{|c|c|c|c|c|c|c|c|}
\hline$*$ & 0 & $\mathrm{x}$ & $\mathrm{y}$ & $\mathrm{z}$ & 1 & $\mathrm{a}$ & $\mathrm{b}$ \\
\hline 0 & 0 & 0 & 0 & 0 & 0 & $\mathrm{a}$ & $\mathrm{a}$ \\
\hline $\mathrm{x}$ & $\mathrm{x}$ & 0 & 0 & 0 & 0 & $\mathrm{a}$ & $\mathrm{a}$ \\
\hline $\mathrm{y}$ & $\mathrm{y}$ & $\mathrm{y}$ & 0 & $\mathrm{y}$ & 0 & $\mathrm{a}$ & $\mathrm{a}$ \\
\hline $\mathrm{z}$ & $\mathrm{z}$ & $\mathrm{z}$ & $\mathrm{z}$ & 0 & 0 & $\mathrm{a}$ & $\mathrm{a}$ \\
\hline 1 & 1 & 1 & 1 & $\mathrm{y}$ & 0 & $\mathrm{a}$ & $\mathrm{a}$ \\
\hline $\mathrm{a}$ & $\mathrm{a}$ & $\mathrm{a}$ & $\mathrm{a}$ & $\mathrm{a}$ & $\mathrm{a}$ & 0 & 0 \\
\hline $\mathrm{b}$ & $\mathrm{b}$ & $\mathrm{a}$ & $\mathrm{a}$ & $\mathrm{a}$ & $\mathrm{a}$ & $\mathrm{y}$ & 0 \\
\hline
\end{tabular}

Tables 2.3

\begin{tabular}{|c|c|c|c|c|c|c|c|}
\hline$\circ$ & 0 & $x$ & $y$ & $z$ & 1 & $a$ & $b$ \\
\hline 0 & 0 & 0 & 0 & 0 & 0 & $a$ & $a$ \\
\hline$x$ & $x$ & 0 & 0 & 0 & 0 & $a$ & $a$ \\
\hline$y$ & $y$ & $y$ & 0 & $y$ & 0 & $a$ & $a$ \\
\hline$z$ & $z$ & $z$ & $z$ & 0 & 0 & $a$ & $a$ \\
\hline 1 & 1 & 1 & $z$ & 1 & 0 & $a$ & $a$ \\
\hline$a$ & $a$ & $a$ & $a$ & $a$ & $a$ & 0 & 0 \\
\hline$b$ & $b$ & $b$ & $a$ & $b$ & $a$ & $x$ & 0 \\
\hline
\end{tabular}

Tables 2.4

Then $(A, \leqslant, *, \circ, 0)$ is a pseudo-BCI algebra.

Proposition 1. [19] In a pseudo-BCI algebras $A$ the following hold:

$\left(p_{1}\right) x \leq 0 \Rightarrow x=0$.

$\left(p_{2}\right) x \leq y \Rightarrow z * y \leq z * x$ and $z \circ y \leq z \circ x$.

$\left(p_{3}\right) x \leq y, y \leq z \Rightarrow x \leq z$.

$\left(p_{4}\right)(x * y) \circ z=(x \circ z) * y$.

$\left(p_{5}\right) x * y \leq z \Leftrightarrow x \circ z \leq y$.

$\left(p_{6}\right)(x * y) *(z * y) \leq x * z,(x \circ y) \circ(z \circ y) \leq x \circ z$.

$\left(p_{7}\right) x \leq y \Rightarrow x * z \leq y * z, x \circ z \leq y \circ z$. 
$\left(p_{8}\right) x * 0=x=x \circ 0$.

$\left(p_{9}\right) x *(x \circ(x * y))=x * y, x \circ(x *(x \circ y))=x \circ y$.

Proposition 2. [19] In a pseudo-BCI algebra $A$ the following holds for all $x, y, z \in A$ :

(i) $0 *(x \circ y) \leq y \circ x$.

(ii) $0 \circ(x * y) \leq y * x$.

(iii) $0 *(x * y)=(0 \circ x) \circ(0 * y)$.

(iv) $0 \circ(x \circ y)=(0 * x) *(0 \circ y)$.

Definition 3. [19] An element a of a pseudo-BCI algebra $A$ is called a pseudo-atom if for every $x \in A, x \leq a$ implies $x=a$.

The set of all pseudo-atoms of a pseudo-BCI algebra $A$ is denoted by $M(A)$. Obviously, $0 \in M(A)$.

Proposition 3. Let $A$ be a pseudo-BCI algebra and $a \in A$. The following conditions are equivalent:

(1) a is a pseudo-atom of $A$;

(2) $y *(y \circ a)=a($ or $y \circ(y * a)=a)$ for all $y \in A$;

(3) $y *(y \circ(a * x))=a * x($ or $y \circ(y *(a \circ x))=a \circ x)$ for all $x, y \in A$.

Proof. (1) $\Rightarrow(2)$. By $I_{2}, y *(y \circ a) \leq a$. Since $a$ is a pseudo-atom of $A$, we have $y *(y \circ a)=a$.

(2) $\Rightarrow(3)$ Obviously.

$(3) \Rightarrow(1)$ It follows from Proposition 3.6 of [19].

By Proposition 3, we have $x *(x \circ a)=x \circ(x * a)=a$ for all $a \in M(A)$ and $x \in A$.

Corollary 1. Let $A$ be a pseudo-BCI algebra. Then for all $a \in M(A)$ and $x \in A$, we have $a * x \in M(A)$ and $a \circ x \in M(A)$.

Proof. Let $a \in M(A)$ and $x \in A$. By Proposition 3.8(3), we have $y *(y \circ(a * x))=a * x$ for all $y \in A$. Using Proposition 3.8(2), we get that $a * x$ is a pseudo-atom of $A$, that is $a * x \in M(A)$. Similarly we can prove $a \circ x \in M(A)$.

Let $A$ be a pseudo-BCI algebra. For $a \in M(A)$, define $V(a)=\{x \in A \mid a \leq x\}$. V(a) is called a branch of $A$. Obviously $a \in V(a)$.

Proposition 4. Let $A$ be a pseudo-BCI algebra, $a, b \in M(A)$ and $a \neq b$. Then $V(a) \cap$ $V(b)=\emptyset$.

Proof. Assume $V(a) \cap V(b) \neq \emptyset$, then there is $x \in V(a) \cap V(b)$. Hence $a \leq x$ and $b \leq x$. It follows that $(b *(b \circ a)) \circ(b *(b \circ x)) \leq(b \circ x) *(b \circ a) \leq a \circ x=0$. So $(b *(b \circ a)) \circ(b *(b \circ x))=0$. Hence $b *(b \circ a) \leq(b *(b \circ x))=b$. Since $b \in M(A)$, we have $b *(b \circ a)=b$. Note that $b=(b *(b \circ a)) \leq a$. Similarly $a \leq b$. By Definition 3.1, we have $a=b$. It is a contradiction, hence $V(a) \cap V(b)=\emptyset$. 
Proposition 5. Let $A$ be a pseudo-BCI algebra and $x, y \in A$. If $x \leq y$, then $x, y$ are in the same branch of $A$.

Proof. Assume that $x \in V(a)$ and $y \in V(b)$ for some $a, b \in M(A)$ and $a \neq b$. Then $a \leq x \leq y$. Hence $y \in V(a)$ and so $y \in V(a) \cap V(b)$, a contradiction with Proposition 4.

Proposition 6. Let $A$ be a pseudo-BCI algebra and $x \in V(a)$ for some $a \in M(A)$. Then $0 *(0 \circ x)=a$ and $0 \circ(0 * x)=a$.

Proof. Since $0 *(0 \circ x) \leq x$, we have $0 *(0 \circ x) \in V(a)$ by Proposition 5. Hence $a \leq 0 *(0 \circ x)$. On the other hand, we have $(0 *(0 \circ x)) \circ a=(0 \circ a) *(0 \circ x)=$ $((a * x) \circ a) *(0 \circ x)=((a \circ a) * x) *(0 \circ x)=(0 * x) *(0 * x)=0$. Therefore $0 *(0 \circ x) \leq a$. This shows that $0 *(0 \circ x)=a$. Similarly we can prove $0 \circ(0 * x)=a$.

Proposition 7. Let $A$ be a pseudo-BCI algebra. Then for any $x \in A, 0 *(0 \circ x) \in M(A)$ and $0 \circ(0 * x) \in M(A)$.

Proof. Let $x \in A$. In order to prove $0 \circ(0 * x) \in M(A)$, we assume $y \leq 0 \circ(0 * x)$. Then $y \circ(0 \circ(0 * x))=0$. By $\left(p_{4}\right)$ and $\left(p_{9}\right)$ of Proposition 3.3, we have

$(0 \circ(0 * x)) * y=(0 * y) \circ(0 * x)$

$=((y \circ(0 \circ(0 * x))) * y) \circ(0 * x)$

$=((y * y) \circ((0 \circ(0 * x)))) \circ(0 * x)$

$=(0 \circ((0 \circ(0 * x)))) \circ(0 * x)$.

By Proposition $2(\mathrm{iv}), 0 \circ((0 \circ(0 * x)))=(0 * 0) *(0 \circ(0 * x))=0 *(0 \circ(0 * x))=0 * x$. Hence $(0 \circ(0 * x)) * y=(0 \circ((0 \circ(0 * x)))) \circ(0 * x)=(0 * x) \circ(0 * x)=0$. This shows that $0 \circ(0 * x) \leq y$ and hence $y=0 \circ(0 * x)$. Similarly we can prove $0 *(0 \circ x) \in M(A)$.

Corollary 2. Let $A$ be a pseudo-BCI algebra. Then for any $x \in A,(0 \circ x) \in M(A)$ and $(0 * x) \in M(A)$.

Proof. Since $0 * x=0 *(0 \circ(0 * x))$ and $0 \circ x=0 \circ(0 *(0 \circ x))$, we have $0 * x \in M(A)$ and $0 \circ x \in M(A)$ by Proposition 7 .

By Propositions 6 and 7 , we have $0 *(0 \circ x)=0 \circ(0 * x) \in M(A)$ for all $x \in A$. Denote $a_{x}=0 *(0 \circ x)=0 \circ(0 * x)$, for $x \in A$. Then $a_{x} \in M(A)$ and $x \in V\left(a_{x}\right)$.

Using above arguments we can get the structure of a pseudo-BCI algebra.

Theorem 1. Let $A$ be a pseudo-BCI algebra. Then $\{V(a) \mid a \in M(A)\}$ forms a partition of $A$, that is, $A=\cup_{a \in M(A)} V(a)$ and $V(a) \cap V(b)=\emptyset$ for all $a, b \in M(A)$ and $a \neq b$.

\section{Local bounded pseudo-BCI algebras}

Let $A$ be a pseudo-BCI algebra. For $a \in M(A)$, if there is an element $1_{a} \in V(a) \backslash\{a\}$ such that for all $x \in V(a), x \leq 1_{a}$, then $1_{a}$ is called the local unit of $V(a)$. Note that $1_{a}$ is unique. 
Definition 4. Let $A$ be a pseudo-BCI algebra. If for every $a \in M(A), V(a)$ has a local unit, then $A$ is called a local bounded pseudo-BCI algebra. For convenience we denote it by lbp-BCI algebra.

Note that the pseudo-BCI algebras given in Examples 1 and 2 are local bounded pseudo-BCI algebras. In Examples $1, M(A)=\{0, a\}, V(0)=\{0, u, v, w, t, 1\}, 1_{0}=0$, $V(a)=\{a, b\}, 1_{a}=b$. In Examples 2, $M(A)=\{0, a\}, V(0)=\{0, x, y, z, 1\}, 1_{0}=0$, $V(a)=\{a, b\}, 1_{a}=b$.

In the following, $A$ shall mean a lbp-BCI algebra unless otherwise specified.

We define two negations, ${ }^{-}$and ${ }^{\sim}$, as follows: for $a \in M(A)$ and $x \in V(a)$, $x^{-} \doteq 1_{a} * x, x^{\sim} \doteq 1_{a} \circ x$.

Proposition 8. For all $x, y \in A$, we have

(1) $x^{-\sim} \leq x, x^{\sim-} \leq x$.

(2) $x \leq y \Rightarrow y^{-} \leq x^{-}, y^{\sim} \leq x^{\sim}$.

(3) $x^{-}=x^{-\sim-}, x^{\sim}=x^{\sim-\sim}$.

Proof. (1) By $\left(I_{2}\right)$ of Definition 1, we have $x^{-\sim} \leq x$ and $x^{\sim-} \leq x$.

(2) Let $x \leq y$, then $x, y \in V(a)$ for some $a \in M(A)$. Hence $\left(1_{a} \circ y\right) *\left(1_{a} \circ x\right) \leq x \circ y=0$, and so $\left(1_{a} \circ y\right) *\left(1_{a} \circ x\right)=0$. It follows that $1_{a} \circ y \leq 1_{a} \circ x$, or $y^{\sim} \leq x^{\sim}$. Similarly we can prove $y^{-} \leq x^{-}$.

(3) By (1), we have $x^{\sim-} \leq x$. Replace $x$ by $x^{-}$, we get $x^{-\sim-} \leq x^{-}$. On the other hand, $x^{-\sim} \leq x$ implies $x^{-} \leq x^{-\sim-}$ by (2). So $x^{-}=x^{-\sim-}$. Similarly we can prove $x^{\sim}=x^{\sim-\sim}$.

Let $A$ be a pseudo-BCI algebra. For any $x, y \in A$, define $x \wedge_{1} y \doteq y \circ(y * x)$, $x \wedge_{2} y \doteq y *(y \circ x)$.

Proposition 9. In $A$ the following properties hold:

(1) $a_{x} \wedge_{1} x=x \wedge_{1} a_{x}=a_{x}$ and $a_{x} \wedge_{2} x=x \wedge_{2} a_{x}=a_{x}$ for all $x \in A$.

(2) $x \leq y$ implies $y \wedge_{1} x=x$ and $y \wedge_{2} x=x$.

(3) $x \wedge_{1} x=x$ and $x \wedge_{2} x=x$.

(4) If $x_{1} \leq x_{2}$, then $x_{1} \wedge_{1} y \leq x_{2} \wedge_{1} y$ and $x_{1} \wedge_{2} y \leq x_{2} \wedge_{2} y$.

Proof. (1) By Proposition 3, we have $a_{x} \wedge_{1} x=x \circ\left(x * a_{x}\right)=a_{x}$ since $a_{x} \in M(A)$. Note that for $x \in V\left(a_{x}\right)$, we get $x \wedge_{1} a_{x}=a_{x} \circ\left(a_{x} * x\right)=a_{x} \circ 0=a_{x}$. So we shows that $a_{x} \wedge_{1} x=x \wedge_{1} a_{x}=a_{x}$. Similarly we can prove $a_{x} \wedge_{2} x=x \wedge_{2} a_{x}=a_{x}$ for all $x \in A$.

(2) Let $x \leq y$. Then $y \wedge_{1} x=x \circ(x * y)=x \circ 0=x$ and $y \wedge_{2} x=x *(x \circ y)=x * 0=x$.

(3) We have $x \wedge_{1} x=x \circ(x * x)=x$ and $x \wedge_{2} x=x *(x \circ x)=x$.

(4) Let $x_{1} \leq x_{2}$. Note that $\left(x_{1} \wedge_{1} y\right) *\left(x_{2} \wedge_{1} y\right)=\left(y \circ\left(y * x_{1}\right)\right) *\left(y \circ\left(y * x_{2}\right)\right) \leq$ $\left(y * x_{2}\right) \circ\left(y * x_{1}\right) \leq x_{1} * x_{2}=0$. We get $x_{1} \wedge_{1} y \leq x_{2} \wedge_{1} y$. Similarly we can prove $x_{1} \wedge_{2} y \leq x_{2} \wedge_{2} y$.

Proposition 10. In $A$ the following properties hold for all $a \in M(A)$ and $x, y \in V(a)$ :

(1) $x \wedge_{1} y^{-\sim}=x^{-\sim} \wedge_{1} y^{-\sim}$ and $x \wedge_{2} y^{\sim-}=x^{\sim-} \wedge_{2} y^{\sim-}$.

(2) $x \wedge_{1} y^{\sim}=x^{-\sim} \wedge_{1} y^{\sim}$ and $x \wedge_{2} y^{-}=x^{\sim-} \wedge_{2} y^{-}$. 
Proof. (1) Using Proposition 1, we have $y^{-\backsim} * x=\left(1_{a} \circ\left(1_{a} * y\right)\right) * x=\left(1_{a} * x\right) \circ\left(1_{a} * y\right)=$ $\left(1_{a} *\left(1_{a} \circ\left(1_{a} * x\right)\right)\right) \circ\left(1_{a} * y\right)=\left(1_{a} \circ\left(1_{a} * y\right)\right) *\left(1_{a} \circ\left(1_{a} * x\right)\right)=y^{-\backsim} * x^{-\sim}$.

Thus $x \wedge_{1} y^{-\sim}=y^{-\sim} \circ\left(y^{-\sim} * x\right)=y^{-\sim} \circ\left(y^{-\backsim} * x^{-\sim}\right)=x^{-\sim} \wedge_{1} y^{-\backsim}$.

(2) By Proposition 8 and (1), we get

$x \wedge_{1} y^{\sim}=x \wedge_{1}\left(y^{\sim}\right)^{-\sim}=x^{-\sim} \wedge_{1}\left(y^{\sim}\right)^{-\sim}=x^{-\sim} \wedge_{1} y^{\sim}$.

Proposition 11. In $A$ the following properties hold for all $x, y \in A$ :

$y *\left(x \wedge_{1} y\right)=y * x$ and $y \circ\left(x \wedge_{2} y\right)=y \circ x$.

Proof. By Proposition 1, we have $y *\left(x \wedge_{1} y\right)=y *\left(y \circ(y * x)=y * x\right.$ and $y \circ\left(x \wedge_{2} y\right)=$ $y \circ(y *(y \circ x))=y \circ x$.

Proposition 12. Let $a \in M(A)$. If $x, y \in V(a)$, then $x * y \in V(0)$ and $x \circ y \in V(0)$.

Proof. Using Proposition 2 and 6 , we get $0 \circ(0 *(x * y))=0 \circ((0 \circ x) \circ(0 * y))=$ $(0 *(0 \circ x)) *(0 \circ(0 * y))=a * a=0$. Since by $\left(I_{2}\right) 0 \circ(0 *(x * y)) \leq x * y$, we have $0 \leq x * y$, and so $x * y \in V(0)$. Similarly we can prove $x \circ y \in V(0)$.

Proposition 13. In $A$ the following properties hold for all $a \in M(A), x, y \in V(a)$ :

(1) $x \wedge_{1} y\left(y \wedge_{1} x\right)$ is a lower bound of $\{x, y\}$.

(2) $x \wedge_{2} y\left(y \wedge_{2} x\right)$ is a lower bound of $\{x, y\}$.

Proof. By Definition 3.1, we have $x \wedge_{1} y=y \circ(y * x) \leq x$. Moreover by Proposition 12, $y * x \in V(0)$ and so $0 \circ(y * x)=0$, and $(y \circ(y * x)) * y=(y * y) \circ(y * x)=0 \circ(y * x)=0$. It follows that $x \wedge_{1} y=y \circ(y * x) \leq y$. Similarly we can get that $\left(y \wedge_{1} x\right)$ is also a lower bound of $\{x, y\}$.

(2) Similar to the proof of (1).

Definition 5. (1) If for all $a \in M(A)$ and $x, y \in V(a), x \wedge_{1} y=y \wedge_{1} x$, we call $A$ to be $a$ local $\wedge_{1}$-commutative pseudo-BCI algebra.

(2) If for all $a \in M(A)$ and $x, y \in V(a), x \wedge_{2} y=y \wedge_{2} x$, we call $A$ to be a local $\wedge_{2}$ commutative pseudo-BCI algebra.

(3) If $A$ is local $\wedge_{1}$-commutative and local $\wedge_{2}$-commutative, we call $A$ to be local commutative.

Proposition 14. (1) If $A$ is local $\wedge_{1}$-commutative, then $\left(V(a), \wedge_{1}\right)$ forms a lower similattice for all $a \in M(a)$.

(2) If $A$ is local $\wedge_{2}$-commutative, then $\left(V(a), \wedge_{2}\right)$ forms a lower similattice for all $a \in$ $M(a)$.

Proof. (1) It needs only to prove that $x \wedge_{1} y$ is the greatest lower bound of $\{x, y\}$ for all $a \in M(A)$ and $x, y \in V(a)$. Assume that $m$ is a lower bound of $\{x, y\}$. We have

$m *\left(x \wedge_{1} y\right)=(m \circ(m * y)) *(y \circ(y * x))=\left(y \wedge_{1} m\right) *(y \circ(y * x))=\left(m \wedge_{1} y\right) *(y \circ(y * x))=$ $(y \circ(y * m)) *(y \circ(y * x)) \leq(y * x) \circ(y * m) \leq m * x=0$,

and so $m \leq\left(x \wedge_{1} y\right)$. 
(2) Similar to the proof of (1).

For a lbp-BCI algebra $A$, we can define the following operations in $V(a)$, $x \vee_{1} y=1_{a} \circ\left(\left(1_{a} * x\right) \wedge_{1}\left(1_{a} * y\right)\right)$, $x \vee_{2} y=1_{a} *\left(\left(1_{a} \circ x\right) \wedge_{2}\left(1_{a} \circ y\right)\right)$, for all $a \in M(A)$ and for all $x, y \in V(a)$.

Proposition 15. Let $A$ be a lbp-BCI algebra.

(1) If $A$ is local $\wedge_{1}$-commutative, then $\left(V(a), \wedge_{1}, \vee_{1}\right)$ forms a lattice for all $a \in M(a)$.

(2) If $A$ is local $\wedge_{2}$-commutative, then $\left(V(a), \wedge_{2}, \vee_{2}\right)$ forms a lattice for all $a \in M(a)$.

Proof. (1) Let $a \in M(A)$ and $x, y \in V(a)$. Since $A$ is local $\wedge_{1}$-commutative, then $x=x \circ\left(x * 1_{a}\right)=1_{a} \circ\left(1_{a} * x\right) \leq 1_{a} \circ\left(\left(1_{a} * x\right) \wedge_{1}\left(1_{a} * y\right)\right)=x \vee_{1} y$. Similarly we can prove $y \leq x \vee_{1} y$.

If $z \geq x$ and $z \geq y$, then $z \in V(a), 1_{a} * x \geq 1_{a} * z$ and $1_{a} * y \geq 1_{a} * z$. By Proposition 14 , we have $1_{a} * z \leq\left(1_{a} * x\right) \wedge_{1}\left(1_{a} * y\right)$. Therefore $x \vee_{1} y=1_{a} \circ\left(\left(1_{a} * x\right) \wedge_{1}\left(1_{a} * y\right)\right) \leq$ $1_{a} \circ\left(1_{a} * z\right)=z \circ\left(z * 1_{a}\right)=z$. It follows that $x \vee_{1} y$ is the least upper bound of $\{x, y\}$.

Applying Proposition 14, we get $\left(V(a), \wedge_{1}, \vee_{1}\right)$ forms a lattice.

(2) Similar to the proof of (1).

Definition 6. Let $A$ be a pseudo-BCI algebra. (1) If for all $x, y \in A, x \wedge_{1} y=y \wedge_{1} x$, we call $A$ to be $\wedge_{1}$-commutative.

(2) If for all $x, y \in A, x \wedge_{2} y=y \wedge_{2} x$, we call $A$ to be $\wedge_{2}$-commutative.

(3) If $A$ is $\wedge_{1}$-commutative and $\wedge_{2}$-commutative, we call $A$ to be sup-commutative.

The following result shows that $\wedge_{1}$-commutative $\left(\wedge_{2}\right.$-commutative $)$ pseudo-BCI algebras must be pseudo-BCK algebras.

Proposition 16. Let $A$ be a pseudo-BCI algebra. Then the following are equivalent:

(1) $A$ is $\wedge_{1}$-commutative $\left(\wedge_{2}\right.$-commutative).

(2) $A$ is a $\wedge_{1}$-commutative $\left(\wedge_{2}\right.$-commutative) pseudo-BCK algebra.

Proof. (1) $\Rightarrow(2)$. Let $A$ be $\wedge_{1}$-commutative. Then for any $a \in M(A)$, we have $a \wedge_{1} 0=0 \wedge_{1} a$. Note that $a \wedge_{1} 0=0 \circ(0 * a)=a$ by Proposition 6 and $0 \wedge_{1} a=a \circ(a * 0)=0$. This shows that $a=0$, that is $A=V(0)$. Thus $A$ is a $\wedge_{1}$-commutative pseudo-BCK algebra. Similarly we can prove the result for case of $\wedge_{2}$-commutative.

$(2) \Rightarrow(1)$. It is straightforward.

Proposition 17. [15] If $A$ is a sup-commutative pseudo-BCK algebra, then $\wedge_{1}=\wedge_{2}$.

By Proposition 16 and 17, we can get a characterization of sup-commutative pseudoBCI algebras.

Proposition 18. Let $A$ be a pseudo-BCI algebra. Then the following are equivalent:

(1) $A$ is a sup-commutative pseudo-BCI algebra.

(2) $A$ is a sup-commutative pseudo-BCK algebra. 


\section{States on local bounded pseudo-BCI algebras}

Definition 7. Let $A$ be a lbp-BCI algebra. A Bosbach state on $A$ is a function $s: A \rightarrow$ $[0,1]$ such that the following conditions hold:

(1) $s(x)+s(y * x)=s(y)+s(x * y)$, for all $x, y \in A$,

(2) $s(x)+s(y \circ x)=s(y)+s(x \circ y)$, for all $x, y \in A$,

(3) $s(a)=1$ and $s\left(1_{a}\right)=0$ where $a \in M(A)$ and $1_{a}$ is the local unit of $V(a)$.

Example 3. Consider the local bounded pseudo-BCI algebra A given in Example 1. Define the function $s: A \rightarrow[0,1]$ by $s(0)=1, s(u)=1, s(v)=1, s(w)=1, s(t)=1, s(1)=$ $0, s(a)=1, s(b)=0$. Then $s$ is a unique Bosbach state on $A$.

Example 4. Consider the local bounded pseudo-BCI algebra A given in Example 2. Define a function $s: A \rightarrow[0,1]$ as follows: $s(0)=1, s(x)=\alpha, s(y)=\beta, s(z)=\gamma, s(1)=0, s(a)=$ $1, s(b)=0$. Using $s(u)+s(v * u)=s(v)+s(u * v)$, taking $u=x, v=1, u=y, v=1$ and $u=z, v=1$, respectively, we get $\alpha=1, \beta=1, \gamma=0$. On the other hand, taking $u=z$, $v=1$ in $s(u)+s(v \circ u)=s(v)+s(u \circ v)$, we get $\gamma+0=0+1$, so $0=1$ which is a contradiction. Hence $A$ does not admit a Bosbach state.

Proposition 19. Let $A$ be a lbp-BCI algebra and $s$ a Bosbach state on $A$. Then the following properties hold for all $x, y \in A$ :

(1) If $x \leq y$, then $s(y * x)=1+s(y)-s(x)=s(y \circ x)$ and $s(y) \leq s(x)$.

(2) If $x, y$ are in same branch, then $s\left(x \wedge_{1} y\right)=s\left(y \wedge_{1} x\right), s\left(x \wedge_{2} y\right)=s\left(y \wedge_{2} x\right)$.

(3) If $x, y$ are in same branch, then $s\left(x \wedge_{1} y^{-\sim}\right)=s\left(x^{-\sim} \wedge_{1} y^{-\sim}\right), s\left(x \wedge_{2} y^{\sim-}\right)=s\left(x^{\sim-} \wedge_{2}\right.$ $\left.y^{\sim-}\right)$.

(4) If $x, y$ are in same branch, then $s\left(x^{-\sim} \wedge_{1} y\right)=s\left(x \wedge_{1} y^{-\sim}\right), s\left(x^{\sim-} \wedge_{2} y\right)=s\left(x \wedge_{2} y^{\sim-}\right)$.

(5) $s\left(x^{-\sim}\right)=s(x)=s\left(x^{\sim-}\right)$.

(6) $s\left(x^{-}\right)=1-s(x)=s\left(x^{\sim}\right)$.

Proof. (1) Let $x \leq y$. It follows from Definition 5.1 that $s(y * x)=1+s(y)-s(x)=$ $s(y \circ x)$. Moreover $s(x)-s(y)=1-s(y * x) \geq 0$ and hence $s(y) \leq s(x)$.

(2) By Proposition 1, we have $y * x=y *\left(x \wedge_{1} y\right)$. Since $x, y$ are in same branch, then $x \wedge_{1} y \leq x, y$ by proposition 13. By property (1), we have $s(y * x)=s\left(y *\left(x \wedge_{1} y\right)\right)=$ $1+s(y)-s\left(x \wedge_{1} y\right)$ and $s(x * y)=s\left(x *\left(y \wedge_{1} x\right)\right)=1+s(x)-s\left(y \wedge_{1} x\right)$. Using condition (1) from Definition 7 we get $s\left(x \wedge_{1} y\right)=s\left(y \wedge_{1} x\right)$. Similarly we can prove $s\left(x \wedge_{2} y\right)=s\left(y \wedge_{2} x\right)$.

(3) It follows from Proposition 10.

(4) It follows from (2) and (3).

(5) For $x \in A$, there is $a \in M(A)$ such that $x \in V(a)$. Note that $x^{-\sim}=x \wedge_{1} 1_{a}$. By (2), we have $s\left(x^{-\sim}\right)=s\left(x \wedge_{1} 1_{a}\right)=s\left(1_{a} \wedge_{1} x\right)=s\left(x \circ\left(x * 1_{a}\right)\right)=s(x)$. In a similar way, we can prove $s(x)=s\left(x^{\sim-}\right)$.

(6) By (1), we have $s\left(x^{-}\right)=s\left(1_{a} * x\right)=1+s\left(1_{a}\right)-s(x)=1-s(x)$. In a similar way we can get $s\left(x^{\sim}\right)=1-s(x)$. 
Proposition 20. Let $A$ be a lbp-BCI algebra and $s$ be a Bosbach state on $A$. Then the following properties hold for all $a \in M(A)$ and $x, y \in V(a)$ :

(1) $s\left(y * x^{-\sim}\right)=s\left(y^{-\sim} * x\right), s\left(y \circ x^{\sim-}\right)=s\left(y^{\sim-} \circ x\right)$.

(2) $s\left(y^{-\sim} * x\right)=s\left(x^{-} \circ y^{-}\right)=s\left(y^{-\sim} * x^{-\sim}\right)=s\left(y * x^{-\sim}\right)$, $s\left(y^{\sim-} \circ x\right)=s\left(x^{\sim} * y^{\sim}\right)=s\left(y^{\sim-} \circ x^{\sim-}\right)=s\left(y \circ x^{\sim-}\right)$.

(3) $s\left(y^{-\sim} * x^{\sim}\right)=s\left(y * x^{\sim}\right), s\left(y^{\sim-} \circ x^{-}\right)=s\left(y \circ x^{-}\right)$.

Proof. (1) Note that $s\left(y * x^{-\sim}\right)+s\left(y \circ\left(y * x^{-\sim}\right)\right)=s(y)+s\left(\left(y * x^{-\sim}\right) \circ y\right)$, or $s\left(y * x^{-\sim}\right)+s\left(x^{-\sim} \wedge_{1} y\right)=s(y)+s\left(\left(y * x^{-\sim}\right) \circ y\right)$. By Proposition 19(4), we have $s\left(y * x^{-\sim}\right)+s\left(x \wedge_{1} y^{-\sim}\right)=s(y)+s\left(\left(y * x^{-\sim}\right) \circ y\right)=s(y)+s\left((y \circ y) * x^{-\sim}\right)=s(y)+s\left(0 * x^{-\sim}\right)$. Using Corollary 2, we get $0 * x^{-\sim} \in M(A)$, and so $s\left(0 * x^{-\sim}\right)=1$. Thus $s\left(y * x^{-\sim}\right)=$ $s(y)+1-s\left(x \wedge_{1} y^{-\sim}\right)=1-s\left(x \wedge_{1} y^{-\sim}\right)+s\left(y^{-\sim}\right)=s\left(\left(y^{-\sim} * x\right) \circ y^{-\sim}\right)-s\left(x \wedge_{1} y^{-\sim}\right)+s\left(y^{-\sim}\right)=$ $s\left(y^{-\sim} * x\right)$.

Similarly we can prove $s\left(y \circ x^{\sim-}\right)=s\left(y^{\sim-} \circ x\right)$.

(2) By $\left(p_{4}\right)$ we have $s\left(y^{-\sim} * x\right)=s\left(\left(1_{a} \circ\left(1_{a} * y\right)\right) * x\right)=s\left(\left(1_{a} * x\right) \circ\left(1_{a} * y\right)\right)=$ $s\left(x^{-} \circ y^{-}\right)$. Moreover we have $s\left(y^{-\sim} * x^{-\sim}\right)=s\left(\left(1_{a} \circ\left(1_{a} * y\right)\right) *\left(\left(1_{a} \circ\left(1_{a} * x\right)\right)\right)=\right.$ $s\left(\left(1_{a} \circ\left(\left(1_{a} \circ\left(1_{a} * x\right)\right)\right) \circ\left(1_{a} * y\right)\right)=s\left(x^{-\sim-} \circ y^{-}\right)=s\left(x^{-} \circ y^{-}\right)\right.$by Proposition 8. Using (1) we can get $s\left(y^{-\sim} * x\right)=s\left(x^{-} \circ y^{-}\right)=s\left(y^{-\sim} * x^{-\sim}\right)=s\left(y * x^{-\sim}\right)$. Similarly we have $s\left(y^{\sim-} \circ x\right)=s\left(x^{\sim} * y^{\sim}\right)=s\left(y^{\sim-} \circ x^{\sim-}\right)=s\left(y \circ x^{\sim-}\right)$.

(3) By Proposition 5.4(4) we get

$s\left(y^{-\sim} * x^{\sim}\right)=s\left(y^{-\sim}\right)+s\left(\left(y^{-\sim} * x^{\sim}\right) \circ y^{-\sim}\right)-s\left(y^{-\sim} \circ\left(y^{-\sim} * x^{\sim}\right)\right)=s(y)+1-s\left(x^{\sim} \wedge_{1}\right.$ $\left.y^{-\sim}\right)=s(y)+1-s\left(x^{\sim-\sim} \wedge_{1} y\right)=s(y)+1-s\left(x^{\sim} \wedge_{1} y\right)=s\left(y \circ x^{\sim}\right)$.

Similarly we can get $s\left(y^{\sim-} \circ x^{-}\right)=s\left(y \circ x^{-}\right)$.

Proposition 21. Let $A$ be a lbp-BCI algebra and $s$ be a Bosbach state on $A$. Then for all $a \in M(A)$ and $x, y \in V(a), s(y * x)=1-s\left(x \wedge_{1} y\right)+s(y)$ and $s(y \circ x)=1-s\left(x \wedge_{2} y\right)+s(y)$.

Proof. Let $a \in M(A)$ and $x, y \in V(a)$. Note that $x \wedge_{1} y \leq x, y$ and $x \wedge_{2} y \leq x, y$. By $19(1)$, we have $s(y * x)=s\left(y *\left(x \wedge_{1} y\right)\right)=1-s\left(x \wedge_{1} y\right)+s(y)$ and $s(y \circ x)=s\left(y \circ\left(x \wedge_{2} y\right)\right)=$ $1-s\left(x \wedge_{2} y\right)+s(y)$.

The following results are important for our study.

Proposition 22. Let $A$ be a lbp-BCI algebra and $s$ be a Bosbach state on A. Then for all $a \in M(A)$ and $x, y \in V(a)$, we have

(1) $s\left(x \wedge_{1} y\right)=s\left(x \wedge_{2} y\right)$.

(2) $s(x * y)=s(x \circ y)$.

Proof. (1) First we prove the equality for $x \leq y$.

By Propositions 19(2) and 9(2), we have $s\left(x \wedge_{1} y\right)=s\left(y \wedge_{1} x\right)=s(x)$ and $s\left(x \wedge_{2} y\right)=$ $s\left(y \wedge_{2} x\right)=s(x)$, that is $s\left(x \wedge_{1} y\right)=s\left(x \wedge_{2} y\right)$.

Now assume that $x$ and $y$ are arbitrary elements of $V(a)$, where $a \in M(A)$. Using Propositions 19(2) again and first part of the proof, we have $s\left(x \wedge_{1} y\right)=s\left(x \wedge_{1}\left(x \wedge_{1} y\right)\right)=$ $s\left(\left(x \wedge_{1} y\right) \wedge_{1} x\right)=s\left(\left(x \wedge_{1} y\right) \wedge_{2} x\right) \leq s\left(y \wedge_{2} x\right)=s\left(x \wedge_{2} y\right)$.

Dually, we can prove $s\left(x \wedge_{2} y\right) \leq s\left(x \wedge_{1} y\right)$. Hence $s\left(x \wedge_{1} y\right)=s\left(x \wedge_{2} y\right)$. 
(2) It follows from Proposition 21 and the first equation.

Consider the real interval $[0,1]$ of reals equipped with the Eukasiewicz implication $\rightarrow_{\mathrm{E}}$ defined by

$$
x \rightarrow_{\mathrm{E}} y=\min \{1-x+y, 1\} \text {, for all } x, y \in[0,1] .
$$

Definition 8. Let $A$ be a lbp-BCI algebra. A state-morphism on $A$ is a function $m: A \rightarrow$ $[0,1]$ such that:

(SM1) $m(a)=0, m\left(1_{a}\right)=1$ for all $a \in M(A)$.

(SM2) $m(y * x)=m(y \circ x)=m(x) \rightarrow_{L} m(y)$, for all $x, y \in A$.

Proposition 23. Let $A$ be a lbp-BCI algebra. Then every state-morphism on $A$ is a Bosbach state on $A$.

Proof. It is similar to the proof of [[4], Proposition 3.9].

Proposition 24. Let $A$ be a lbp-BCI algebra. A Bosbach state $m$ on $A$ is a state-morphism if and only if $m\left(x \wedge_{1} y\right)=\min \{m(x), m(y)\}$ for all $x, y \in A$, or equivalently, $m\left(x \wedge_{2} y\right)=$ $\min \{m(x), m(y)\}$ for all $x, y \in A$.

Proof. It is similar to the proof of [[4], Proposition 3.10].

Let $A$ be a lbp-BCI algebra and $s$ be a Bosbach state on $A$. Define a set $\operatorname{Ker}(s):=$ $\{x \in A \mid s(x)=1\}$. $\operatorname{Ker}(s)$ is called the kernel of $s$ on $A$.

Definition 9. Let $A$ be a pseudo BCI algebra and I be a nonempty subset of $A$. If I satisfies the following conditions:

(1) $0 \in I$,

(2) $x \in I$ and $y * x \in I$ (or $y \circ x \in I$ ) imply $y \in I$ for all $x, y \in A$,

$I$ is called a pseudo ideal of $A$, simply called an ideal of $A$.

Let $I$ be a pseudo ideal of a pseudo BCI algebra $A$. If $I$ satisfies $0 * x \in I$ and $0 \circ x \in I$, we call $I$ a closed pseudo ideal of $A$. If $I$ satisfies $x * y \in I$ if and only if $x \circ y \in I$, we call $I$ a normal pseudo ideal of $A$. If $I$ satisfies $x * y \in I$ if and only if $x \circ y \in I$ for all $a \in M(A), x, y \in V(a)$, we call $I$ a local normal pseudo ideal of $A$.

Proposition 25. Let $A$ be a lbp-BCI algebra and s be a Bosbach state on A. Then Ker $(s)$ is a closed and local normal proper ideal of $A$.

Proof. Obviously, $0 \in \operatorname{Ker}(s)$ and $1 \notin \operatorname{Ker}(s)$.

Assume that $x, y * x \in \operatorname{Ker}(s)$. Then we have $1=s(x)$ and $s(y * x)=1$. It follows from Definition 5.1 that $s(y)=s(x)+s(y * x)-s(x * y)=2-s(x * y) \geq 1$ and thus $s(y)=1$. Hence $y \in \operatorname{Ker}(s)$. This shows that $\operatorname{Ker}(s)$ is a proper ideal of $A$. For any $x \in A$, we have $0 * x \in M(A)$ and $0 * x \in M(A)$ by Corollary 2. Hence $s(0 * x)=1$ and $s(0 \circ x)=1$. It follows that $0 * x \in \operatorname{Ker}(s)$ and $0 \circ x \in \operatorname{Ker}(s)$. This shows that $I$ is a closed pseudo ideal of $A$. By Proposition 22, we can get that $A$ is local normal. 
Theorem 2. Let $A$ be a pseudo BCI algebra and I be a pseudo ideal of A. Define a binary relation " $\sim$ " on $A$ by $x \sim y$ if and only if $x * y, y * x \in I$ if and only if $x \circ y, y \circ x \in I$. Then $\sim$ is a congruence relation on $A$. Denote $C_{x}=\{y \in A \mid x \sim y\}$. Define $C_{x} * C_{y}=C_{x * y}$ and $C_{x} \circ C_{y}=C_{x \circ y}$. Denote $A / I=\left\{C_{x} \mid x \in A\right\}$. Then $\left(A / I, *, \circ, C_{0}\right)$ is a pseudo BCI algebra. If $I$ is a closed pseudo ideal of $A$, then $C_{0}=I$.

Proof. Obviously $\sim$ is reflexive and symmetric. Now we prove that it is transitive. Let $x \sim y$ and $y \sim z$. Then $x * y, y * z \in I$. By $\left(I_{1}\right),(x * z) \circ(x * y) \leq y * z$, thus $x * z \in I$. Similarly we can prove $z * x \in I$. This shows that $x \sim z$ and hence $\sim$ is transitive. Thus it is an equivalent relation on $A$. We also can show that $\sim$ is a congruence relation on $A$ and omit it. Denote $A / I=\left\{C_{x} \mid x \in A\right\}$. Then binary operations "*" and "o" on $A / I$ are well-defined. Moreover we can show that $(A / I, *, \circ)$ satisfies $I_{1}-I_{5}$ in Definition 3.1. It follows that $\left(A / I, *, \circ, C_{0}\right)$ is a pseudo BCI algebra.

Finally we assume that $I$ is a closed pseudo ideal of $A$. Then for $x \in I$, we have $0 * x \in I$ and $x * 0=x \in I$. Hence $x \sim 0$, that is, $x \in C_{0}$. Therefore $C_{0}=I$.

Proposition 26. Let $s$ be a Bosbach state on a lbp-BCI algebra $A$ and $K=\operatorname{ker}(s)$. Then we have the following.

(1) $x / K \leq y / K$ iff $s(x * y)=1$ iff $s(x \circ y)=1$, where $x / K=\{y \in A \mid y \sim x\}$ for all $x \in A$.

(2) For all $a \in M(A)$ and all $x, y \in V(a)$, we have that $x / K \leq y / K$ iff $s\left(y \wedge_{1} x\right)=s(x)$ iff $s\left(y \wedge_{2} x\right)=s(x)$.

(3) $x / K=y / K$ iff $s(x * y)=s(y * x)=1$ iff $s(x \circ y)=s(y \circ x)=1$.

(4) For all $a \in M(A)$ and all $x, y \in V(a), x / K=y / K$ iff $s(x)=s(y)=s\left(x \wedge_{1} y\right)$ iff $s(x)=s(y)=s\left(x \wedge_{2} y\right)$.

(5) $\left(A / K, \leq, *, \circ, 0 / K, 1_{0} / K\right)$ is a bounded pseudo-BCK algebra where $1_{0}$ is the unit of $V(0)$.

(6) The mapping $\tilde{s}: A / K \rightarrow[0,1]$ defined by $\tilde{s}(x / K):=s(x)(x \in A)$ is a Bosbach state on $A / K$.

Proof. (1) By Theorem 2, we know that $(A / K, \leq, *, \circ, 0 / K)$ is a pseudo-BCI algebra. Note that $x / K \leq y / K$ iff $x / K * y / K=(x * y) / K=0 / K$ iff $x * y \in K$ iff $s(x * y)=1$. Similarly, $x / K \leq y / K$ iff $x / K \circ y / K=(x \circ y) / K=0 / K$ iff $x \circ y \in K$ iff $s(x \circ y)=1$.

(2) Let $a \in M(A)$ and $x, y \in V(a)$. As $s(x * y)=1-s\left(y \wedge_{1} x\right)+s(x)$ by Proposition 21 , we get $x / K \leq y / K$ iff $s\left(y \wedge_{1} x\right)=s(x)$. Similarly, we have $x / K \leq y / K$ iff $s\left(y \wedge_{2} x\right)=s(x)$.

(3) It follows easily from (1).

(4) It follows easily from (2).

(5) First we prove $M(A / K)=\{0 / K\}$. Let $x / K \leq 0 / K$. By (1), $s(x * 0)=1$. Note that $0 * x \in M(A)$, then we have $s(0 * x)=1$. By $(3), x / K=0 / K$. Thus $0 / K \in M(A / K)$.

Conversely let $x / K \in M(A / K)$. Obviously $(0 *(0 * x)) / K \leq x / K$. Hence $(0 *(0 *$ $x)) / K=x / K$. Since for any $a \in M(A), s(a * 0)=s(0 * a)=1$, we have $0 / K=a / K$. Thus $x / K=(0 *(0 * x)) / K=0 / K$. This shows that $M(A / K)=\{0 / K\}$, and hence $(A / K, \leq, *, \circ, 0 / K)$ is a pseudo-BCK algebra.

Now we prove that $1_{0} / K$ is the greatest element of $A / K$. First we claim $1_{0} / K=1_{a} / K$ for all $a \in M(A)$. Note that $s\left(1_{0}\right)+s\left(1_{a} * 1_{0}\right)=s\left(1_{a}\right)+s\left(1_{0} * 1_{a}\right)$ and $s\left(1_{0}\right)=s\left(1_{a}\right)=0$ 
by Definition 7 , we have $s\left(1_{a} * 1_{0}\right)=s\left(1_{0} * 1_{a}\right)$. Moreover $s\left(1_{a} * 1_{0}\right)+s\left(a \circ\left(1_{a} * 1_{0}\right)\right)=$ $s(a)+s\left(\left(1_{a} * 1_{0}\right) \circ a\right)$ by Definition 7 . By Corollary $1, a \circ\left(1_{a} * 1_{0}\right) \in M(A)$, and so $s\left(a \circ\left(1_{a} * 1_{0}\right)\right)=1$. Since $\left(1_{a} * 1_{0}\right) \circ a=\left(1_{a} \circ a\right) * 1_{0}$ and $1_{a} \circ a \in V(0)$ by Proposition 12 , we have $s\left(\left(1_{a} * 1_{0}\right) \circ a\right)=s\left(\left(1_{a} \circ a\right) * 1_{0}\right)=s(0)=1$. Hence $s\left(1_{a} * 1_{0}\right)=1$. By $(3), 1_{0} / K=1_{a} / K$ for all $a \in M(A)$. Let $x / K \in A / K$. Then $x / K \leq 1_{(0 *(0 \circ x))} / K=1_{0} / K$. This shows that $1_{0} / K$ is the greatest element of $A / K$. It follows that $\left(A / K, \leq, *, \circ, 0 / K, 1_{0} / K\right)$ is a bounded pseudo BCK algebra.

(6) The fact that $\tilde{s}$ is a well-defined Bosbach state on $A / K$ is now straightforward.

Definition 10. Let $A$ be a lbp-BCI algebra. Then

(1) $A$ is called good if $x^{-\sim}=x^{\sim-}$ for all $x \in A$.

(2) $A$ is with the condition $(p D N)$ if $x^{-\sim}=x^{\sim-}=x$ for all $x \in A$.

Proposition 27. Let $s$ be a Bosbach state on a bounded pseudo-BCI algebra $A$ and let $K=\operatorname{ker}(s)$. For every element $x \in A$, we have $x^{-\sim} / K=x / K=x^{\sim-} / K$, that is, $A / K$ satisfies the $(p D N)$ condition.

Proof. It is similar to the proof of [[4], Proposition 3.14].

Remark 2. Let $s$ be a Bosbach state on a pseudo-BCI algebra A. According to the proof of Proposition 27, we have $s\left(x * x^{-\sim}\right)=1=s\left(x * x^{\sim-}\right)$ and $s\left(x \circ x^{-\sim}\right)=1=s\left(x \circ x^{\sim-}\right)$.

Theorem 3. Let $A$ be a lbp-BCI algebra, $s$ be a Bosbach state on $A$ and $K=\operatorname{ker}(s)$. Then $A / K$ is $\wedge_{1}$-commutative as well as $\wedge_{2}$-commutative. In addition, $A / K$ is a $\wedge$-semilattice and good.

Proof. It is similar to the proof of [[4], Proposition 3.16].

Proposition 28. ([4]) Let $A$ be a good pseudo-BCK algebra. We define a binary operation $\otimes$ on $A$ by $x \otimes y:=y^{-\sim} * x^{\sim}$. For all $x, y \in A$, the following hold:

(1) $x \otimes y=x^{\sim-} \circ y^{-}$.

(2) $x \otimes y \leq x, y$.

(3) $x \otimes 1=1 \otimes x=x^{\sim-}$.

(4) $x \otimes 0=0 \otimes x=0$.

(5) $(x \otimes y)^{-\sim}=x \otimes y=x^{-\sim} \otimes y^{-\sim}$.

$(6) \otimes$ is associative.

An MV-algebra is an algebra $\left(A, \oplus,^{-}, 0\right)$ of type $(2,1,0)$ such that (i) $\oplus$ is commutative and associative, (ii) $x \oplus 0=x$, (iii) $x \oplus 0^{-}=0^{-}$,(iv) $x^{--}=x$,(v) $y \oplus\left(y \oplus x^{-}\right)^{-}=$ $x \oplus\left(x \oplus y^{-}\right)^{-}$. If we define $x * y=x \circ y=y^{-} \oplus x$, then $(A, *, \circ, 1,0)$ is a bounded pseudo-BCK algebra.

An MV-state on an MV-algebra $A$ is a mapping $s: A \rightarrow[0,1]$ such that $s(1)=1$ and $s(a \oplus b)=s(a)+s(b)$ whenever $a \odot b=0$. Every MV-algebra admits at least one MV-state, and due to [17], every MV-state on $A$ coincides with a Bosbach state on the BCK algebra $A$ and vice versa. 
We note that the radical, $\operatorname{Rad}(A)$, of an MV-algebra $A$ is the intersection of all maximal ideals of $A([7])$.

Proposition 29. ([9]). In any $M V$-algebra $A$ the following conditions are equivalent:

(a) $\operatorname{Rad}(A)=0$.

(b) $n x \leq x^{-}$for all $n \in N$ implies $x=0$.

(c) $n x \leq y^{-}$for all $n \in N$ implies $x \wedge y=0$.

(d) $n x \leq y$ for all $n \in N$ implies $x \odot y=x$, where $n x=x_{1} \oplus \cdots x_{n}$ with $x_{1}=\cdots=x_{n}=x$.

Remark 3. An $M V$-algebra $A$ is archimedean in the sense of [9] if it satisfies the condition (b) of Proposition 29 and $A$ is archimedean in Belluces sense [1] if it satisfies the condition

(d) of Proposition 29. By Proposition 29 the two definitions of archimedean MV-algebras are equivalent.

Theorem 4. Let $s$ be a Bosbach state on a lbp-BCI algebra $A$ and let $K=\operatorname{Ker}(s)$. Then $\left(A / K, \oplus,^{-}, 0 / K\right)$, where $a / K \oplus b / K=\left(b * a^{-}\right) / K$ and $(a / K)^{-}=a^{-} / K$, is an archimedean $M V$-algebra and the map $\hat{s}(a / K):=s(a)$ is an $M V$-state on this $M V$-algebra.

Proof. It is similar to the proof of [[4], Theorem 3.20].

By Theorem $3, A / K$ is a good pseudo-BCK algebra that is a $\wedge$-semilattice and $\tilde{s}$ on $A / K$ is a Bosbach state such that $\operatorname{Ker}(\tilde{s})=\{0 / K\}$. Due to [[20], Proposition 3.4.7], $(A / K) / \operatorname{Ker}(\tilde{s})$ is term-equivalent to an MV-algebra that is archimedean and $\tilde{s}$ is an MVstate on it. Since $A / K=(A / K) / \operatorname{Ker}(\tilde{s})$, the same is true also for $A / K$, and this proves the theorem.

In the following, we give properties of state-morphisms on lbp-BCI algebras.

Lemma 1. Let $A$ be a lbp-BCI algebra and $m$ be a state-morphism on $A$. Then we have the following.

(1) $m\left(y^{-\sim} * x^{\sim}\right)=\min \{m(x)+m(y), 1\}$, for all $a \in M(A)$ and $x, y \in V(a)$.

(2) $m\left(x^{\sim-} \circ y^{-}\right)=\min \{m(x)+m(y), 1\}$, for all $a \in M(A)$ and $x, y \in V(a)$.

Proof. Assume that $m$ is a state-morphism on $A$, so it is a Bosbach state on $A$. By Propositions 19 and 20, for for all $a \in M(A)$ and $x, y \in V(a)$, we have $m\left(y^{-\sim} * x^{\sim}\right)=$ $m\left(y * x^{\sim}\right)=m\left(x^{\sim}\right) \rightarrow_{\mathrm{E}} m(y)=m(x)^{\sim} \rightarrow_{\mathrm{E}} m(y)=\min \left\{1-m(x)^{\sim}+m(y), 1\right\}=$ $\min \{m(x)+m(y), 1\}$. Similarly we can prove $m\left(x^{\sim-} \circ y^{-}\right)=\min \{m(x)+m(y), 1\}$, for all $a \in M(A)$ and $x, y \in V(a)$.

Proposition 30. Let $A$ be a lbp-BCI algebra and $s$ be a Bosbach state on $A$. Then the following are equivalent:

(1) $s$ is a state-morphism.

(2) $\operatorname{ker}(s)$ is a maximal ideal of $A$.

Proof. It is similar to the proof of [[4], Proposition 3.22]. 
Lemma 2. Let $m$ be a state-morphism on a lbp-BCI algebra $A$ and $K=k e r(m)$. Then

(1) $a / K \leq b / K$ if and only if $m(a) \leq m(b)$,

(2) $a / K=b / K$ if and only if $m(a)=m(b)$.

Proof. It is similar to the proof of [[4], Lemma 3.23].

Proposition 31. Let $A$ be a lbp-BCI algebra and $m_{1}, m_{2}$ be two state-morphisms on $A$ such that $\operatorname{ker}\left(m_{1}\right)=\operatorname{ker}\left(m_{2}\right)$. Then $m_{1}=m_{2}$.

Proof. By Proposition 23, $m_{1}$ and $m_{1}$ are two Bosbach states on $A$. Since $\operatorname{ker}\left(m_{1}\right)=$ $\operatorname{ker}\left(m_{2}\right)$, we have $A / \operatorname{ker}\left(m_{1}\right)=A / \operatorname{ker}\left(m_{2}\right)$. By the proof of Proposition 30, we have that $A / \operatorname{ker}\left(m_{1}\right)$ is in fact an MV-subalgebra of the MV-algebra of the real interval [0,1]. But $\operatorname{ker}\left(\hat{m}_{1}\right)=0 / K=\operatorname{ker}\left(\hat{m}_{2}\right)$. Hence, by [[11], Proposition 4.5], $\hat{m}_{1}=\hat{m}_{2}$, consequently, $m_{1}=m_{2}$.

Let $A$ be a lbp-BCI algebra. We say that a Bosbach state $s$ is extremal if for any $0<\lambda<1$ and for any two Bosbach states $s_{1}, s_{2}$ on $A, s=\lambda s_{1}+(1-\lambda) s_{2}$ implies $s_{1}=s_{2}$. Summarizing previous characterizations of state-morphisms, we have the following result.

Theorem 5. Let $s$ be a Bosbach state on a lbp-BCI algebra A. Then the following are equivalent:

(1) $s$ is an extremal Bosbach state.

(2) $s\left(x \wedge_{1} y\right)=\max \{s(x), s(y)\}$ for all $x, y \in A$.

(3) $s\left(x \wedge_{2} y\right)=\max \{s(x), s(y)\}$ for all $x, y \in A$.

(4) $s$ is a state-morphism.

(5) $\operatorname{ker}(s)$ is a maximal ideal.

Proof. It is similar to the proof of [[4], Theorem 3.26].

\section{Conclusions}

Until now, the states on unbounded algebraic structures have been studied for Hilbert algebras and integral residuated lattices in [2] and [6], respectively.

In this paper, we first study state theory on non-bounded algebraic structures, and introduce a notion of state on pseudo-BCI algebras. In order to adapt a state to pseudo-BCI algebras, we first discuss the structure of pseudo-BCI algebras, which can be decomposed in to the union of it's branches. Note that for all $a \in M(A)$ and $a \neq 0, V(a)$ is not a BCK-algebra, hence the structure of pseudo-BCI algebras is different from the structure of pseudo-BCK algebras. Therefore it is valuable to study state theory on pseudo-BCI algebras. Moreover we introduce a notion of local bounded pseudo-BCI algebras and set up the theory of states on such algebraic structure. We also introduce a notion of state-morphisms on local bounded pseudo-BCI algebras and discuss the relations between Bosbach states and state-morphisms. By use of state's theory, we discuss the relation between pseudo$\mathrm{BCI}$ algebras and MV-algebras. In the next work, we will consider the following problem: satisfying what apposite conditions a local bounded pseudo-BCI algebra admits a Bosbach state? 


\section{Acknowledgements}

This research is partially supported by a grant of National Natural Science Foundation of China (11571281,61602359), China Postdoctoral Science Foundation (2015M582618), China 111 Project (B16037).

\section{References}

[1] L P Belluce. Semisimple and complete MV-algebras. Algebra Univ., 29:1-9, 1992.

[2] C Buşneag. States on Hilbert algebras. Studia Logica, 94:177-188, 2010.

[3] C C Chang. Algebraic analysis of many valued logics. Transactions of the American Mathematical society, 88(2):467-490, 1958.

[4] L C Ciungu and A Dvurečenskij. Measures, states and de Finetti maps on pseudo-BCK algebras. Fuzzy Sets and Systems, 161(22):2870-2896, 2010.

[5] L C Ciungu, A Dvurečenskij and M Hycko. State BL-algebras. Soft Computing, 15(4):619-634, 2011.

[6] L C Ciungu. Relative negations in non-commutative fuzzy structures. Soft Computing, 18:15-33, 2014.

[7] R Cignoli, I M L DOttaviano and D Mundici. Algebraic Foundations of Many-valued Reasoning. Kluwer Academic Publishers, Dordrecht, 2000.

[8] W A Dudek and Y B Jun. Pseudo-BCI algebras. East Asian Math. J., 24:187-190, 2008.

[9] A Dvurečenskij and S Pulmannová. New Trends in Quantum Structures. Kluwer Academic Publishers, Dordrecht, Boston, London, 2000.

[10] A Dvurečenskij. Measures and states on BCK-algebras. Atti Del seminario matematico e fisico universita di modena, 47:511-528, 1996.

[11] A Dvurečenskij. States on pseudo MV-algebras. Studia Logica, 68:301-327, 2001.

[12] A Dvurečenskij and T Vetterlein. Algebras in the positive cone of po-groups Order, 19:127-146, 2002.

[13] G Georgescu and A Iorgulescu. Pseudo-BCKalgebras: An extension of BCKalgebras. in:Proc.DMTCS01: Combinatorics, Computability and Logic, Springer, London, pp.97$114,2001$.

[14] P Hájek. Metamathematics of Fuzzy Logic. Kluwer Academic Publishers, Dordrecht, 1998. 
[15] A Iorgulescu. Pseudo-Iséki algebras. Connection with pseudo-BL algebras. J. Multiple-Valued Logic Soft Comput., 3-4:263-308, 2005.

[16] A Iorgulescu. Algebras of Logic as BCK-algebras. Academy of Economic Studies Bucharest, Editura, 2008.

[17] K Iséki. An algebra related with a propositional calculus. Proc. Japan Acad., 42:26-29, 1966.

[18] K Iséki. On BCI-algebras. Math. Sem. Notes Kobe Univ., 8(1):125-130, 1980.

[19] Y B Jun, H K Kim and J Neggers. On pseudo-BCI ideals of pseudo-BCI algebras. Matematiqki Vesnik, 58:39-46, 2006.

[20] J Kuhr. Pseudo-BCK algebras and related structures. Univerzita Palackého v Olomouci, 2007.

[21] L Lianzhen. States on finite monoidal t-norm based algebras. Information Sciences, 181:1369-1393, 2011.

[22] J Meng and Y B Jun. BCK-algebras. Kyungmoon Sa, Seoul, 1994.

[23] D Mundici. Averaging the truth-value in Łukasiewicz logic. Studia Logica, 55(1):113127, 1995.

[24] D Mundici. Interpretation of $\mathrm{AFC}^{*}$-algebras in Lukasiewicz sentential calculus. J. Funct. Anal, 65: 15-53, 1986. 